\title{
Localization of Abnormal Conduction Pathways for Tachyarrhythmia Treatment Using Tagged MRI
}

\author{
G.I. Sanchez-Ortiz ${ }^{1}$, M. Sermesant ${ }^{2}$, K.S. Rhode ${ }^{2}$, R. Chandrashekara ${ }^{1}$, \\ R. Razavi ${ }^{2}$, D.L.G. Hill ${ }^{3}$, and D. Rueckert ${ }^{1}$ \\ 1 Imperial College London, U.K \\ 2 King's College London, U.K. \\ 3 University College London, U.K.
}

\begin{abstract}
Tachyarrhythmias are pathological fast heart rhythms often caused by abnormally conducting myocardial areas (foci). Treatment by radio-frequency $(\mathrm{RF})$ ablation uses electrode-catheters to monitor and destroy foci. The procedure is normally guided with x-rays (2D), and thus prone to errors in location and excessive radiation exposure. Our main goal is to provide pre- and intra-operative $3 \mathrm{D}$ MR guidance in XMR systems by locating the abnormal conduction pathways. We address the inverse electro-mechanical relation by using motion in order to infer electrical propagation. For this purpose we define a probabilistic measure of the onset of regional myocardial activation, derived from 3D motion fields obtained by tracking tagged MR sequences with non-rigid registration. Activation isochrones are then derived to determine activation onset.

We also compare regional motion between two different image acquisitions, thus assisting in diagnosing arrhythmia, in follow up of treatment, and in determining whether the ablation was successful. Difference maps of isochrones and other motion descriptors are computed to determine abnormal patterns. Validation was carried out using an electromechanical model of the heart, synthetic data, a cardiac MRI atlas of motion and geometry, MRI data from 6 healthy volunteers (one of them subjected to stress), and an MRI study on a patient with tachyarrhythmia, before and after RF ablation. A pre-operative MRI study on a second patient with tachyarrhythmia was used to test the methodology in a clinical scenario, predicting the abnormally conducting region.
\end{abstract}

\section{Introduction}

Superventricular tachyarrhythmias are pathological fast heart rhythms most commonly caused by an extra electrical propagation pathway (foci) between the atria and ventricles. Treatment by radio-frequency (RF) ablation uses electrodecatheters to monitor endocardial electrical activity. An ablation electrode is then used to apply a RF current, inducing hyperthermia and thus destroying the abnormally conducting areas. The procedure is normally guided with x-rays (2D), and thus prone to errors in location and excessive radiation exposure. 
Our main objective in this work is to provide pre- and intra-operative 3D MR guidance [1,2] in XMR systems (combined X-ray and MRI room) by detecting the onset of regional motion and relating it to the electrical activation pattern. For this purpose we define a probabilistic measure of regional motion activation derived from a 3D motion field extracted by using non-rigid 3D registration of tagged MR (SPAMM) image sequences. Since we address the inverse electromechanical problem, trying to infer time of electrical activation by extracting information from the cardiac motion, we use an electro-mechanical model of the heart to validate these results. Isochrones computed from MR motion are compared between different image acquisitions, and also to those isochrones obtained with the model. A cardiac MR atlas of motion and geometry is also used to validate results in a realistic but relatively noise-free case.

Another objective of this work is to detect changes in regional motion patterns between two different image acquisitions. The purpose of this being the follow up of medical treatment in general, and in particular of patients that have undergone RF ablation. For these patients the method can aid in the identification and localisation of abnormal or changing motion patterns, and also can help determine whether the ablation had the desired effect of regularising cardiac contraction. For validation we use MR images of 6 healthy volunteers (one subjected to stress), synthetic data generated with a cardiac motion simulator of MR images, and pre- and post-intervention MR images on a patient with tachyarrhythmia. Difference maps of isochrones and other motion descriptors are computed on the anatomically meaningfully subdivided myocardium.

In order to test accuracy and feasibility in clinical use, a pre-operative MRI study of a second patient with tachyarrhythmia was used for predicting the abnormally conducting region and results compared against those of three experts.

\section{Methods}

\subsection{Registration for Motion Tracking}

We use a non-rigid registration algorithm [3] to track the motion and deformation of the heart in a sequence of 3D short- and long-axis tagged MR images. The goal of the non-rigid registration is to align each time frame of the tagged MR image sequence with the end-systolic (ES) time frame of the image sequence by maximising the normalised mutual information of both time frames. To model cardiac motion we use a free-form deformation based on cubic B-splines. The output of the registration is a continuous time varying $3 \mathrm{D}$ motion vector field, $\mathbf{F}(\mathbf{p}, t)$, where $\mathbf{F}: \Re^{4} \rightarrow \Re^{3}$ and $\mathbf{p} \in \Re^{3}$ is the spatial coordinate $(x, y, z)$.

\subsection{Coordinate System and Myocardial Segmentation}

A manual segmentation of the myocardium at end-diastole (ED) is used to determine the region of interest (myo) for the registration at time $t=0$. Using $\mathbf{F}$, the myocardial region can be automatically propagated over the entire cardiac cycle. 
In order to be able to compare different image acquisitions, a common (cylindrical) coordinate system based on the left ventricle is defined for each subject, thus avoiding potential misregistration errors due to subject motion between scans. We then express the $\mathbf{F}$ in terms of radial, circumferential and longitudinal directions, and subdivide the myocardium (myo) into small meaningful regions or segments $s$. For the purpose of comparing motion between different scans we use $S=12$ segments (similar to those suggested by the AHA): 4 sections around the $\mathrm{z}$-axis that correspond to septum, lateral, anterior and posterior walls, and 3 sections along the $\mathrm{z}$-axis, corresponding to base, middle region and apex.

\subsection{Differential Motion Descriptors and Changes in Motion Patterns}

Some differential features derived from the motion field $\mathbf{F}(\mathbf{p}, t)$ are useful to describe myocardial non-rigid motion. We write them as the set of functions

$$
F^{m}=F^{m}(\mathbf{p}, t) \text { where } m \in \mu=\{D, R, C, Z, \dot{R}, \dot{C}, \dot{Z}, E, r, c, z, \dot{r}, \dot{c}, \dot{z}\}
$$

and $F^{m}: \Re^{4} \rightarrow \Re$ are defined as the total deformation or displacement $F^{D}=$ $\|\mathbf{F}\|$, the radial, circumferential and longitudinal components of the deformation $\left(F^{R}, F^{C}\right.$ and $\left.F^{Z}\right)$ with respect to the cylindrical coordinate system and their corresponding time derivatives or velocities $\left(F^{\dot{R}}, F^{\dot{C}}\right.$ and $\left.F^{\dot{Z}}\right)$, the magnitude of the strain matrix $F^{E}=\left\|E_{i, j}\right\|$, the radial, circumferential and longitudinal components of the strain $\left(F^{r}, F^{c}\right.$ and $\left.F^{z}\right)$, and their time derivatives $\left(F^{\dot{r}}, F^{\dot{c}}\right.$ and $F^{\dot{z}}$ ), all with respect to the the same cylindrical coordinate system.

The values of $F^{m}(\mathbf{p}, t)$ are first computed for each voxel, and then averaged for each of the myocardial segments $s$, for all time frames during the cardiac cycle:

$$
F^{m}(s, t)=\frac{1}{\int_{\mathbf{p} \in s} d \mathbf{p}} \int_{\mathbf{p} \in s} F^{m}(\mathbf{p}, t) d \mathbf{p} \quad \text { for all regions } s \in \mathbf{m y o} .
$$

In order to evaluate changes in the motion patterns between two data sets $\mathbf{F}_{1}$ and $\mathbf{F}_{2}$, for instance those corresponding to pre- and post-ablation scans, the difference between the two functions $F_{1}^{m}$ and $F_{2}^{m}$ is computed for each segment, integrated over time and normalised using the maximum value of the function for the specific segment. This normalization of the values compensates for the differences in the dynamic behaviour expected in the various regions of the heart (like apex and base for instance). A statistical measure is derived from the above combined quantities [4] and each segment is assigned a measure of motion change and classified as having either no, small or significant changes.

\subsection{Activation Detection}

Although the study of myocardial electrical phenomena such as the excitationcontraction relation, re-entries and patterns occurring inside the myocardium remain open problems for study (see references in [5,6]), in this work we use the underling assumption that we can relate the onset of regional motion, derived from 
the images sequences, to the electrical activation (i.e. using the inverse relation of electro-mechanical coupling). Ideally the onset of regional contraction could be inferred from the motion field simply from strain, but we use a more robust measure because of noise and the relatively low space and time resolution of the images and the extracted motion fields. For this purpose we investigate the subset of differential descriptors $\mathbf{F}^{m}$ where $m \in M=\{R, C, Z, \dot{R}, \dot{C}, \dot{Z}, E, \dot{r}, \dot{c}, \dot{z}\}$.

The first step to characterise the regional motion of the heart during the cardiac cycle is to measure the regional $\left(T_{E S}(s)\right)$ and global $\left(T_{E S}\right)$ end-systolic times, as well as the critical times for each motion descriptor. We therefore define $T_{\text {max }}^{m}(s)=t^{*}$ such that $F^{m}\left(s, t^{*}\right) \geq F^{m}(s, t) \forall t \in\left[0, T_{E S}(s)\right]$ and $T_{\text {min }}^{m}(s)=t^{*}$ such that $F^{m}\left(s, t^{*}\right) \leq F^{m}(s, t) \forall t \in\left[T_{\max }^{m}(s), T_{E S}(s)\right]$. Notice that for $T_{\text {min }}^{m}$ the search interval begins at $T_{\text {max }}^{m}$, i.e. when the maximum value has been reached (it is the late minimum value of $F^{m}$ that will help us define the end-systolic time, not the small values at the beginning of the cycle). Computing these values requires a first (visual) estimate of the end-systolic time, however a short iterative process rapidly provides a better estimate for $T_{E S}(s)$.

In the case of displacement and strain, the end-systolic time is linked to their maximum values, while in the case of velocity and rate of change of strain it corresponds to their minimum values (when the heart has paused its contraction). Therefore,

$$
T_{E S}^{m}(s)=\left\{\begin{array}{l}
T_{\max }^{m}(s) \text { for } m \in\{R, C, Z, E\} \\
T_{\min }^{m}(s) \text { for } m \in\{\dot{R}, \dot{C}, \dot{Z}, \dot{r}, \dot{c}, \dot{z}\}
\end{array}\right.
$$

and combining these times we obtain an estimate that corresponds to the regional time of end-systole: $T_{E S}(s)=\sum_{m \in M} w_{m} T_{E S}^{m}(s)$. The weights $w_{m}$ are normalised (i.e. $\sum_{m \in M} w_{m}=1$ ) and reflect the confidence we have on each of the differential motion descriptors $m$. At present we assign these weights manually, but a statistical measure derived from the data will be used to compute them automatically. In order to obtain a global estimate for end-systolic time for each feature we integrate those values over the entire myocardium: $T_{E S}=\int_{s \in \mathbf{m y o}} T_{E S}(s) d s$. Using the above equations we define a probabilistic measure of the activation for every voxel in the myocardium, at any time during the cardiac cycle:

$$
A(s, t)=\sum_{m \in M} w_{m} \int_{0}^{t} \frac{F^{m}(s, \tau)}{\int_{0}^{T_{m a x}^{m}(s)} F^{m}\left(s, \tau^{\prime}\right) d \tau^{\prime}} d \tau
$$

where we impose $F^{m}(s, t)=0$ if $t>T_{\text {max }}^{m}(s)$ in order to keep the values normalised (notice that some motion descriptors like the velocities and the timederivatives of strain reach their maximum values before end-systole).

The value of $A(s, t)$ monotonically increases from zero to one as we expect every voxel to have been activated by the time the motion descriptors reach the maximum value at time $T_{\max }^{m}(s)$. In order to avoid singularities in the equation we excluded from the computation, and labelled as not active, those voxels that might remain relatively static (i.e. those for which $F^{m}\left(s, T_{\max }^{\operatorname{m}}(s)\right) \approx 0$ ). By integrating over time we obtain an accumulated probability and we can therefore 
set a (percentage) threshold $P$, between 0 and 1 , to define the time $t_{a}$ at which the activation of a segment $s$ takes place. That is, if $A\left(s, t_{a}\right)=P$ then $s$ becomes active for $t=t_{a}$. The activation isochrones are then defined, for a given threshold $P$, as the function $A(s)=t_{a}$, for all $s \in$ myo.

\subsection{Cardiac Motion Simulator for Tagged MRI}

In order to validate the proposed methodology with a controlled case we also implemented and modified a cardiac motion simulator for tagged MRI [7]. The motion simulator is based on a 13-parameter model of left-ventricular motion developed by Arts et al. [8] and is applied to a volume representing the LV that is modeled as a region between two confocal prolate spheres while the imaging process is simulated by a tagged spin-echo imaging equation [9].

A pair of sequences of synthetic tagged LV images was produced in the following manner: first, a 'post-intervention' (normal) sequence computed using the standard model parameters, and secondly, a 'pre-intervention' (abnormal) sequence computed for which the motion parameters were modified in a small region of the myocardium by bringing the contraction slightly forward in time [4].

\section{Results and Discussion}

\subsection{Changes in Regional Motion Patterns}

The detection of changes in motion patterns was evaluated on synthetic data as well as real MR data. In order to test the algorithm when the ground truth is available, results on the 'pre-' and 'post-intervention' sequences of synthetic tagged LV images were compared in two cases, with different parameters and
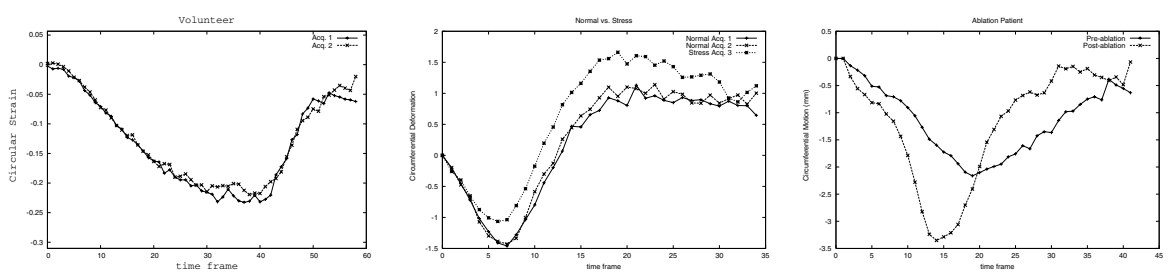

Fig. 1. Time plots of differential motion descriptors. (a) Similar $F^{m}$ curves of a typical myocardial segment obtained from two independent scans of a healthy volunteer demonstrate reproducibility. (b) A myocardial segment of a healthy volunteer, with and without stress. There are no significant changes in the motion pattern between the first two image acquisitions. In the third image acquisition (under stress) a noticeable alteration was detected. (c) A myocardial segment of patient before and after RF ablation. Significant change can be seen in the faster and more pronounced motion of the post-intervention sequence, indicating successful regularisation of the contraction. 
regions of abnormal motion (see Section 2.5). In both cases the abnormal segments were accurately located, while the remaining ones were correctly classified as having no significant change [4. We also acquired MR data from six volunteers. For each of them two separate sets of image sequences were acquired with only a few minutes between the scans. Since no change is expected in these pairs of image acquisitions, this allowed us to verify the reproducibility of the motion fields computed by the algorithm and to test the comparison method against false positive detection. The motion patterns encountered were very similar and no region was classified as having a significant change (Figure 1a).

With another volunteer we acquired three sets of image sequences with only few minutes between the scans. The first two were normal scans as described above, but the third one was acquired while inducing stress on the volunteer by placing one of his feet into a bucket of ice-cold water. This experiment allowed us to compare normal motion patterns against those obtained under stress, and again, to validate the method regarding reproducibility and false positives. No segment showed a significant difference between the two normal acquisitions, but few segments did when compared to the stress acquisition (Figure 10).

Finally, MRI data was acquired from an eight year old patient with acute super-ventricular tachyarrhythmia, before and after RF ablation. The image acquisition and catheter intervention were carried out with an XMR system [1]. Our results confirmed that the motion pattern changed in most parts of the myocardium (visual inspection of the reconstructed 3D surfaces and displacement vectors also showed pronounced changes in the overall contraction pattern), while the largest changes were found in five segments. Examples of the compared motion also show the corrective effect of the intervention (see Figure 15).

\subsection{Activation Detection}

Figure 2 shows results of activation detection obtained for the MR stress study described in Section 3.1. The times of activation of different regions of the myocardium are shown as different colours over the end-diastolic myocardial surface

(a)

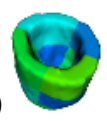

(b)

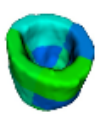

(c)

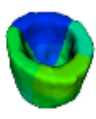

(d)

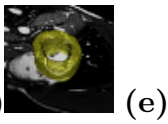

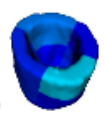

(f)

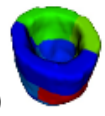

(g)

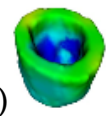

Fig. 2. Isochrones of stress data. Motion-derived activation isochrones computed from two normal MR scans, (a) and (b), and a third one acquired while the volunteer was subjected to stress (c). The anatomical MR image and LV surface skeleton shows the display orientation in (d). Isochrones subtraction maps: the difference between the two normal repetition scan in (e), and the difference between a normal and the stress scan in (f). Isochrones computed from the electro-mechanical model are shown in (g). The colour scales go from blue to red: isochrones maps from 0 to $500 \mathrm{~ms}$, with green approx. $200 \mathrm{~ms}$, and in isochrones subtraction maps from 0 to $100 \mathrm{~ms}$ approx. 
(activation isochrones maps). The first three images in the figure compare the isochrones obtained from the three MR data acquisitions of the same subject: two repetition scans with no changes in between them, and a third scan acquired while the volunteer was subjected to stress. Results of subtracting pairs of isochrones maps are also shown: the difference between the two normal repetition acquisitions, in Figure 2, and the difference between a normal and the stress acquisition, in Figure 2f. We can see that the difference between the isochrones of the two normal acquisitions is small, thus validating the method regarding reproducibility, while on the other hand some larger changes can be appreciated between the isochrones of the normal and the stress scans, thus highlighting the regions that were most affected by stress.

Since we are addressing the problem of inverse electro-mechanical coupling, that is, trying to infer the time of electrical activation by extracting information from the cardiac motion images, we have also used a forward 3D electromechanical model of the heart [5] to validate the activation detection results. The segmentation of the myocardium of a healthy volunteer at end-diastole was used as geometric input for the model. The muscle fiber orientation and the Purkinje network location were fitted to the geometry from a-priori values of the model. Figure $2 \mathrm{~b}$ shows the isochrones values computed using the electromechanical model applied to the subject of the stress study. Good correlation can be seen between these and the isochrones derived from MR motion.

We also used a cardiac atlas of geometry and motion, generated from 3D MR images sequences of 14 volunteers, to test our activation measure in a real-

(a)

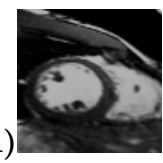

(b)

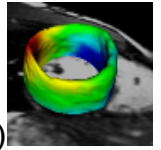

(c)

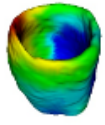

(d)

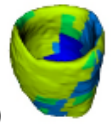

Fig. 3. Isochrones of cardiac atlas. Isochrones were computed for the atlas using both, the electro-mechanical model ((b) and (c)), and the proposed activation measure derived from the motion field $((\mathbf{d}))$. The colour scale goes from blue to red (earliest to latest activation time). The orientation of the left and right ventricle can be seen on the MR images of the subject used as a reference for the atlas $((\mathbf{a})$ and $(\mathbf{b}))$.

(a)

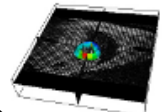

(b)

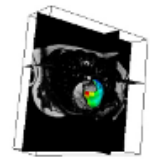

(c)

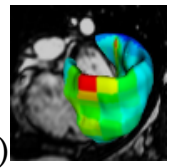

(d)

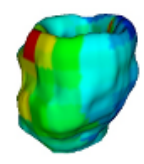

Fig. 4. LV surface with activation times derived from the motion field of a patient with WPW syndrome. The orientation of the left and right ventricles can be seen on the tagged image (a), and in two views ((b) and (c)) of the high resolution anatomical image. In order to highlight the area of earliest motion $(\mathbf{( d )})$, fed by the abnormal conduction pathway, the colour scale in this figure goes from red (earliest) to blue (latest). 
istic but smooth and virtually noise-free data set [10]. For the purpose of comparing activation detection results to those obtained with the high-resolution electro-mechanical model, a larger number of smaller segments was used (also, segments can be very small in this case since there is little noise in the data). Figure 3 compares the isochrones for the atlas computed by both, the electromechanical model, and the proposed activation measure derived from the motion field. Promising agreement can be seen on these results of activation detection.

In order to test the accuracy and feasibility in clinical use, a preoperative MRI study of a second patient with tachyarrhythmia (Wolff-ParkinsonWhite (WPW) syndrome) was processed before the RF ablation, and the location of the abnormal conduction pathway automatically estimated as the region of earliest activation. To increase the accuracy of the estimated position, instead of using 12, we used 120 segments for the LV: 24 subdivisions around the z-axis and 5 from apex to base. The geometric centre of the earliest activated segment was used as the automated estimated position of the pathway (Figure 4).

For the purpose of validation, two experts involved with the patient's RF ablation were separately asked to estimate the location of the abnormal pathway based on careful visual inspection of a 3D anatomical MR image sequence of the patient. This anatomical scan, acquired immediately after the tagged one, had higher time resolution, thus facilitating the visual assessment of the earliest site of motion. With respect to the expert's estimations, the distances (errors) of the automatically estimated position were $5.01 \mathrm{~mm}$ and $5.27 \mathrm{~mm}$. The distance between the expert's positions was under 2mm. Further confirmation of this result came from the analysis of the patient's ECG recordings by a third expert who estimated the location of the pathway to be in the posterior-septum.

\section{Conclusions and Future Work}

Despite current limitations such as distinguishing between epi- and endo-cardial activation patterns, the methodology seems promising for the assessment of intervention results and could also be used for the detection of arrhythmia, ischaemia, regional disfunction, and follow-up studies in general. Because acquisition of tagged images can be carried out in less than 20 minutes, either immediately before the RF ablation or the day before the intervention, the proposed analysis is suitable for clinical practice in guiding and monitoring the effects of ablation on ventricular arrhythmias, with little extra discomfort added to the patient. As has been shown, the error in the location of the abnormal pathway can be as small as $5 \mathrm{~mm}$, as independently confirmed by two experts. In order to account for possible changes in the heart rate between the pre- and post-intervention acquisitions (or for instance, in the case of the stress study where there was a small change in the heart rate), we intend to re-scale one of the image sequences in the time domain, by using the $4 \mathrm{D}$ registration technique described in 10 . 


\section{References}

[1] K.S. Rhode, D.L.G. Hill, P.J. Edwards, J. Hipwell, D. Rueckert, G.I. SanchezOrtiz, S. Hegde, V. Rahunathan, and R. Razavi. Registration and tracking to integrate X-ray and MR images in an XMR facility. IEEE Trans Med Imag, 22(11):1369-78, 2003.

[2] G.I. Sanchez-Ortiz, M. Sermesant, R. Chandrashekara, K.S. Rhode, R. Razavi, D.L.G. Hill, and D. Rueckert. Detecting the onset of myocardial contraction for establishing inverse electro-mechanical coupling in XMR guided RF ablation. In IEEE Int Symp Biomed Imag (ISBI'04), pages 1055-8, Arlington, USA, Apr 2004.

[3] R. Chandrashekara, R. Mohiaddin, and D. Rueckert. Analysis of 3D myocardial motion in tagged MR images using nonrigid image registration. IEEE Trans Med Imag, 23(10):1245-1250, 2004.

[4] G.I.Sanchez-Ortiz, M.Sermesant, K.S.Rhode, R.Chandrashekara, R.Razavi, D.L.G Hill, and D.Rueckert. Detecting and comparing the onset of myocardial activation and regional motion changes in tagged MR for XMR-guided RF ablation. In Functional Imaging and Modeling of the Heart, LNCS 3504, pages 348-58, 2005.

[5] M. Sermesant, K. Rhode, A. Anjorin, S. Hedge, G. Sanchez-Ortiz, D. Rueckert, P. Lambiase, C. Bucknall, D. Hill, and R. Razavi. Simulation of the electromechanical acitvity of the heart using XMR interventional imaging. In MICCAI'04, LNCS 3217, pages 786-94, France, 2004.

[6] E. McVeigh, O. Faris, D. Ennis, P. Helm, and F. Evans. Measurement of ventricular wall motion, epicardial electrical mapping, and myocardial fiber angles in the same heart. In FIMH'01, LNCS 2230, pages 76-82, 2001.

[7] E. Waks, J. L. Prince, and A. S. Douglas. Cardiac motion simulator for tagged MRI. In IEEE Worksh. Math. Meth. Biomed. Imag. Anal., pages 182-91, 1996.

[8] T.Arts, W.Hunter, A.Douglas, A.Muijtjens, and R.Reneman. Description of the deformation of the LV by a kinematic model. Biomech., 25(10):1119-27, 1992.

[9] J. L. Prince and E. R. McVeigh. Motion estimation from tagged MR images. IEEE Transactions on Medical Imaging, 11(2):238-249, June 1992.

[10] D.Perperidis, M.Lorenzo-Valdes, R.Chandrashekara, R.Mohiaddin, G.I.SanchezOrtiz, and D.Rueckert. Building a $4 \mathrm{D}$ atlas of the cardiac anatomy and motion using MR imaging. In IEEE Int. Symp. Biomed. Imag., pages 412-5, USA, 2004. 